Article

\title{
Design, Synthesis and Insecticidal Activity of Novel Phenylurea Derivatives
}

\author{
Jialong Sun ${ }^{\dagger}$ and Yuanming Zhou ${ }^{\dagger} * *$ \\ College of Chemistry and Pharmaceutical Sciences, Qingdao Agricultural University, \\ Qingdao 266109, China; E-Mail: sunjialong6289@163.com \\ $\dagger$ These authors contributed equally to this work. \\ * Author to whom correspondence should be addressed; E-Mail: zym7410@163.com; \\ Tel.: +86-532-8608-0147.
}

Academic Editor: Jean Jacques Vanden Eynde

Received: 28 February 2015 / Accepted: 17 March 2015 /Published: 19 March 2015

\begin{abstract}
A series of novel phenylurea derivatives were designed and synthesized according to the method of active groups linkage and the principle of aromatic groups bioisosterism in this study. The structures of the novel phenylurea derivatives were confirmed based on ESI-MS, IR and ${ }^{1} \mathrm{H}-\mathrm{NMR}$ spectral data. All of the compounds were evaluated for the insecticidal activity against the third instars larvae of Spodoptera exigua Hiibner, Plutella xyllostella Linnaeus, Helicoverpa armigera Hubner and Pieris rapae Linne respectively, at the concentration of $10 \mathrm{mg} / \mathrm{L}$. The results showed that all of the derivatives displayed strong insecticidal activity. Most of the compounds presented higher insecticidal activity against $S$. exigua than the reference compounds tebufenozide, chlorbenzuron and metaflumizone. Among the synthesized compounds, $\mathbf{3 b}, \mathbf{3 d}, \mathbf{3 f}, \mathbf{4 b}$ and $4 \mathrm{~g}$ displayed broad spectrum insecticidal activity.
\end{abstract}

Keywords: phenylurea; diacylhydrazine; metaflumizone; insecticidal activity; synthesis

\section{Introduction}

Benzoylphenyl ureas (BPUs), acting as insect growth regulators (IGRs) and affecting the larval stages of most insects by blocking or inhibiting the synthesis of chitin, have been rapidly developed since the first benzoylphenylurea, diflubenzuron, was commercialized in 1972 [1,2]. BPUs have 
attracted considerable attention for decades for their unique mode of action coupled with a high degree of activity on target pests and low toxicity to non-target organisms [3,4]. More than 20 BPUs have been developed as IGRs, such as chlorfluazuron, flufenoxuron, triflumuron and chlorbenzuron [5].

Diacylhydrazines have been another of the most important classes of insect growth regulators since the discovery of the $N$-tert-butyl- $N, N$ '-diacylhydrazines in the mid-1980s by Rohm and Haas Co. [6,7]. They affect the ecdysone receptor and lead to lethal premature molting. For their unique mode of action with high insecticidal selectivity, lower toxicity to vertebrates and simple structure, diacylhydrazines have attracted considerable attention [8,9]. Among nonsteroidal ecdysone agonists, $N$-tert-butyl- $N$ '-(4-ethylbenzoyl)-3,5-dimethylbenzoylhydrazine (tebufenozide) was the first introduced to market [10,11]. At present, a series of diacylhydrazines, such as methoxyfenozide, halofenozide and chromafenozide have already been widely applied as pesticides in the agrochemical field.

Metaflumizone, which was discovered by Nihon Nohyaku in the early 1990s, is a novel sodium channel blocker insecticide. It belongs to the new class of semicarbazone insecticides and its structure contains an acylhydrazone moiety. It provides good control of most of the economically important lepidopterous pests and other orders pests including Siphonaptera, Diptera, Hymenoptera and Coleoptera. Meanwhile, it presents low risk to non-target organisms as well as humans and the environment. Moreover, insect strains which were resistant to the organophosphates, imidacloprid and carbamates have not showed cross-resistance to metaflumizone. Therefore, metaflumizone has a great potential in Integrated Pest Management(IPM) strategy and resistance management [12-14].

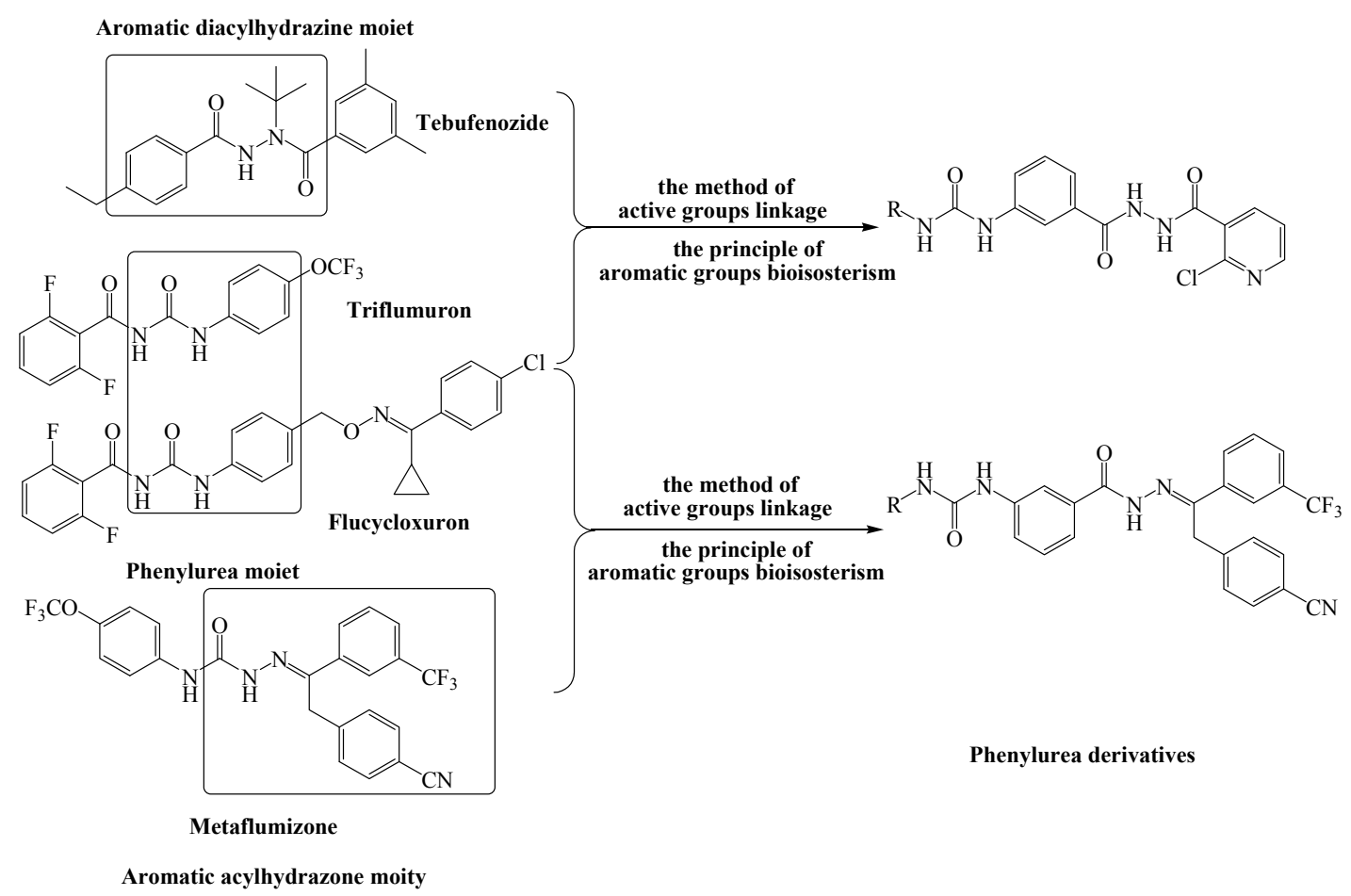

Figure 1. Design of the skeleton of phenylurea derivatives.

An abundance of insects belonging to the order Lepidoptera, such as the diamondback moth (Helicoverpa armigera Hubner), beet armyworm (Spodoptera exigua Hiibner), cabbage caterpillar (Pieris rapae Linne) and cotton bollworm (Plutella xyllostella Linnaeus) etc., are among the most damaging pests for crops all over the world. Because they cause enormous agricultural production 
losses, various insecticides have been used to control Lepidoptera pests. These insecticides brought great benefits, but they have caused negative effects such as toxicity to non-target organisms, including mammals, and environmental pollution. Moreover insecticide resistance has strengthened yearly [15]. Therefore, researchers have to develop novel, low toxicity, highly efficient, friendly environmental insecticides.

In view on the facts above, we sought to incorporate a phenylurea unit with an aromatic diacylhydrazine and acylhydrazone moiety, respectively, according to the method of active groups linkage and the principle of aromatic groups bioisosterism (Figure 1), and eighteen such phenylurea derivatives were then designed and synthesized (Scheme 1). Moreover the new compounds' insecticidal activities against the third instars larvae of diamondback moth, beet armyworm, cabbage caterpillar and cotton bollworm, were evaluated. We expected that by this approach the combination of the critical components could further strengthen the biological activity of phenylurea derivatives.

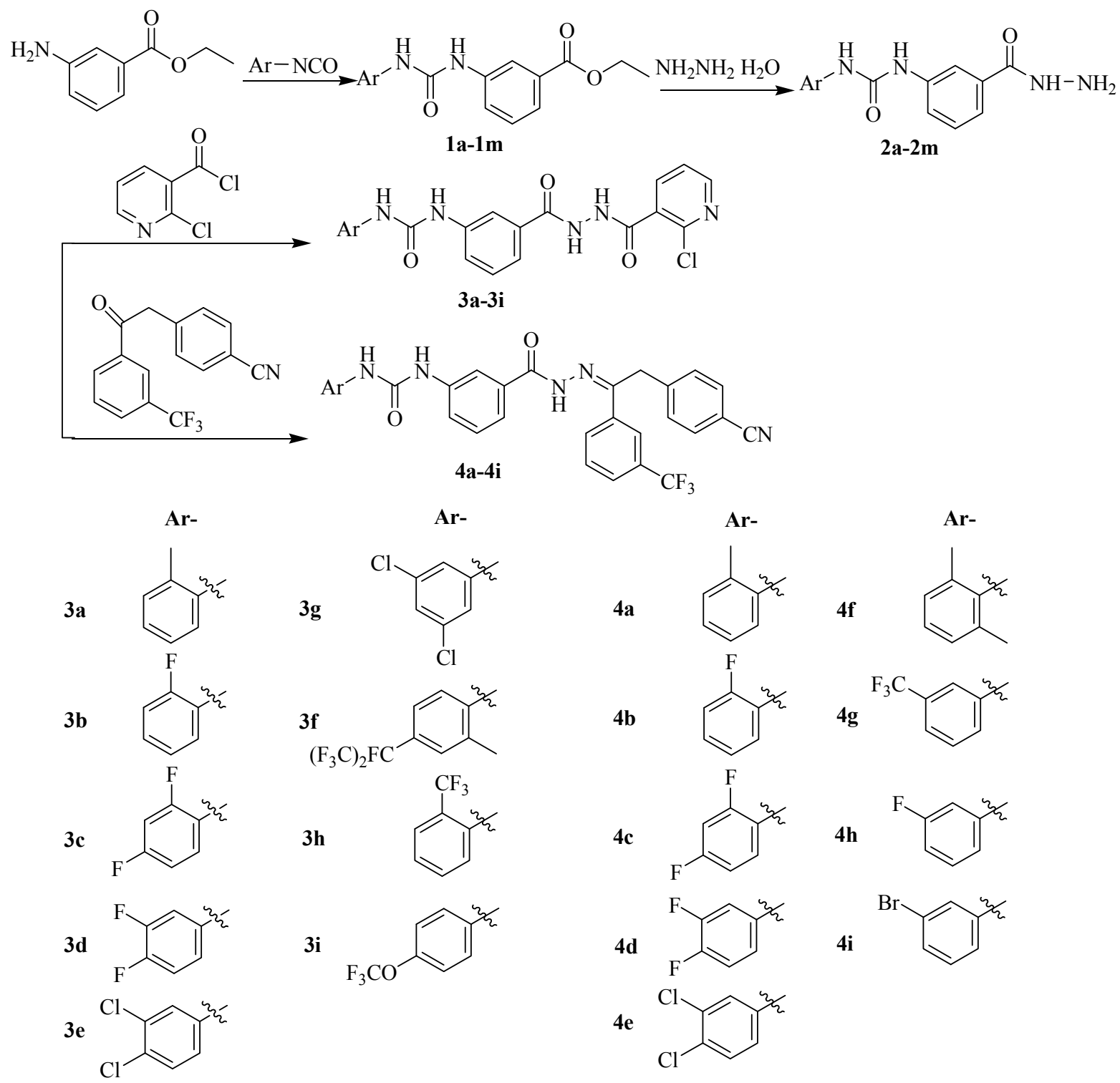

Scheme 1. Synthesis of phenylurea derivatives. 


\section{Results and Discussion}

\subsection{Chemistry}

As indicated in Scheme 1, eighteen phenylurea derivatives $3 \mathbf{a}-3 \mathbf{i}, 4 \mathbf{a}-\mathbf{4 i}$ were successfully synthesized. The raw materials 3-aminobenzoic acid ethyl ester and a substituted phenyl isocyanate (Ar-NCO) were dissolved in toluene and refluxed to give ethyl 3-(3-substituted phenylureido) benzoates $\mathbf{1 a}-\mathbf{1} \mathbf{m}$. Then with alcohol as solvent, 3-(3-substituted phenylureido)benzohydrazides $\mathbf{2 a - 2} \mathbf{m}$ were produced by the reaction of compounds $\mathbf{1 a}-\mathbf{1 m}$ with hydrazine hydrate. Subsequently, compounds $\mathbf{2 a}-\mathbf{2} \mathbf{m}$ reacted with 2-chloronictinoyl chloride in tetrahydrofuran at room temperature to provide phenylurea derivatives, $N^{\prime}$-[3-(3-substituted phenylureido)benzoyl]- $N$-(2-chloro)nicotino-hydrazides 3a-3i. Meanwhile, compounds $\mathbf{2 a - 2 m}$ also reacted with 2-(4-nitrilo)benzyl-1-(3-trifluoromethyl)phenyl ketones in tetrahydrofuran at room temperature to give others phenylurea derivatives, $\mathrm{N}$-substituted phenylureido benzoyl-1-(3-trifluoromethyl)phenyl-2-(4-cyano) phenyl ethanone hydrazones 4a-4i. The structures of all the phenylurea derivatives were confirmed by ${ }^{1} \mathrm{H}-\mathrm{NMR}$, IR, ESI-MS and MP (Scheme 1).

\subsection{Insecticidal Activities}

As displayed in Table 1, all of the phenylurea derivatives $\mathbf{3 a}-\mathbf{3 i}, \mathbf{4 a}-\mathbf{4 i}$ indicated strong insecticidal activities against the third instars larval of beet armyworm at the concentration of $10 \mathrm{mg} / \mathrm{L}$. Most of the synthesized compounds showed higher insecticidal activity than the references chlorbenzuron, tebufenozide and metaflumizone at the $72 \mathrm{~h}$ time point. Among the synthesized compounds, the mortality of the compounds $\mathbf{3 g}, \mathbf{3 i}, \mathbf{4 d}$ and $\mathbf{4 e}$ exceeded $90 \%$ at $72 \mathrm{~h}$. Moreover the mortality of compound $\mathbf{4 g}$ reached $100 \%$. Meanwhile the data of Table 1 shows that the mortality of the compounds indicated a positive correlation with administration time.

Table 1. Insecticidal activities of the phenylurea derivatives against the third instars larval of beet armyworm (Concentration of $10 \mathrm{mg} / \mathrm{L}$ ).

\begin{tabular}{ccccccccc}
\hline \multirow{2}{*}{ Compound } & \multicolumn{3}{c}{ Mortality (\%) } & \multirow{3}{*}{ Compound } & \multicolumn{3}{c}{ Mortality (\%) } \\
\cline { 2 - 4 } \cline { 7 - 9 } & $\mathbf{2 4} \mathbf{h}$ & $\mathbf{4 8} \mathbf{~ h}$ & $\mathbf{7 2} \mathbf{~ h}$ & & $\mathbf{2 4} \mathbf{h}$ & $\mathbf{4 8} \mathbf{~ h}$ & $\mathbf{7 2} \mathbf{~ h}$ \\
\hline $\mathbf{3 a}$ & 12.50 & 45.83 & 83.33 & $\mathbf{4 a}$ & 25.00 & 41.67 & 87.50 \\
$\mathbf{3 b}$ & 8.33 & 37.50 & 87.50 & $\mathbf{4 b}$ & 12.50 & 37.50 & 75.00 \\
$\mathbf{3 c}$ & 4.17 & 37.50 & 83.33 & $\mathbf{4 c}$ & 20.83 & 45.83 & 79.17 \\
$\mathbf{3 d}$ & 12.50 & 45.83 & 87.50 & $\mathbf{4 d}$ & 50.00 & 70.83 & 91.67 \\
$\mathbf{3 e}$ & 16.67 & 50.00 & 87.50 & $\mathbf{4 e}$ & 41.67 & 75.00 & 95.83 \\
$\mathbf{3 f}$ & 8.33 & 41.67 & 87.50 & $\mathbf{4 f}$ & 41.67 & 66.67 & 87.50 \\
$\mathbf{3 g}$ & 20.83 & 66.67 & 95.83 & $\mathbf{4 g}$ & 45.83 & 75.00 & 100.0 \\
$\mathbf{3 h}$ & 8.33 & 41.67 & 83.33 & $\mathbf{4 h}$ & 16.67 & 37.50 & 79.17 \\
$\mathbf{3 i}$ & 16.67 & 62.50 & 91.67 & $\mathbf{4 i}$ & 20.83 & 45.83 & 83.33 \\
Tebufenozide & 4.17 & 20.83 & 41.67 & Chlorbenzuron & 12.50 & 16.67 & 45.83 \\
Metaflumizone & 37.50 & 66.67 & 75.00 & & & & & \\
\hline
\end{tabular}


The data in Table 2 showed the mortality of the phenylurea derivatives $3 \mathbf{a}-\mathbf{3 i}, \mathbf{4 a}-\mathbf{4 i}$ against the third instars larval of diamondback moth (P. xyllostella), cotton bollworm (H. armigera) and cabbage worm ( $P$. rapae) at the concentration of $10 \mathrm{mg} / \mathrm{L}$ after $72 \mathrm{~h}$. The data presented that all the phenylurea derivatives showed strong insecticidal activity against diamondback moth, cotton bollworm and cabbage worm. Among the synthesized compounds, 3b, 3d, 3f, 4b and 4g displayed broad spectrum insecticidal activity.

Table 2. Insecticidal activities of phenylurea derivatives (Concentration of $10 \mathrm{mg} / \mathrm{L}, 72 \mathrm{~h}$ ).

\begin{tabular}{cccc}
\hline \multirow{2}{*}{ Compound } & \multicolumn{3}{c}{ Mortality(\%) } \\
\cline { 2 - 4 } & P. xyllostella & H. armigera & P. rapae \\
\hline 3a & 87.50 & 91.67 & 91.67 \\
$\mathbf{3 b}$ & 91.67 & 95.83 & 95.83 \\
$\mathbf{3 c}$ & 87.50 & 91.67 & 91.67 \\
$\mathbf{3 d}$ & 91.67 & 95.83 & 95.83 \\
$\mathbf{3 e}$ & 91.67 & 87.50 & 95.83 \\
$\mathbf{3 f}$ & 95.83 & 91.67 & 95.83 \\
$\mathbf{3 g}$ & 83.33 & 83.33 & 100.0 \\
$\mathbf{3 h}$ & 83.33 & 87.50 & 91.67 \\
$\mathbf{3 i}$ & 95.83 & 83.33 & 100.0 \\
$\mathbf{4 a}$ & 79.17 & 87.50 & 91.67 \\
$\mathbf{4 b}$ & 91.67 & 95.83 & 95.83 \\
$\mathbf{4 c}$ & 83.33 & 79.17 & 87.50 \\
$\mathbf{4 d}$ & 79.17 & 87.50 & 100.0 \\
$\mathbf{4 e}$ & 75.00 & 87.50 & 100.0 \\
$\mathbf{4 f}$ & 87.50 & 91.67 & 87.50 \\
$\mathbf{4 g}$ & 91.67 & 95.83 & 100.0 \\
$\mathbf{4 h}$ & 91.67 & 87.50 & 87.50 \\
$\mathbf{4 i}$ & 83.33 & 79.17 & 87.50 \\
Tebufenozide & 83.33 & 83.33 & 87.50 \\
Chlorbenzuron & 87.50 & 79.17 & 83.33 \\
Metaflumizone & 66.67 & 37.50 & 75.00 \\
\hline
\end{tabular}

\section{Experimental Section}

\subsection{General Procedures}

${ }^{1} \mathrm{H}-\mathrm{NMR}$ spectra were obtained at $500 \mathrm{MHz}$ using an AM-500 spectrometer in DMSO-d6 solution (Bruker, Karlsruhe, Germany). The melting points were determined on WRS-1A type melting point apparatus (Shanghai, China) and uncorrected. IR spectra were measured on a Nicolet IR-200 spectrophotometer (Thermo Electron, Madison, WI, USA) using KBr disks. A Micromass Q-TOF spectrometer (Waters Crop., Manchester, UK) was used to record HR-ESI-MS data. Pre-coated silica gel plates were used for analytical thin layer chromatography (TLC) and spots were visualized with UV (254 nm).Yields were not optimized. 


\subsection{General Procedure for the Preparation of $\mathbf{1 a - 1 m}$}

3-Aminobenzoic acid ethyl ester $(50 \mathrm{mmol})$ was dissolved in toluene $(30 \mathrm{~mL})$. With ice-water bath cooling, a substituted phenyl isocyanate (Ar-NCO) $(50 \mathrm{mmol})$ dissolved in toluene $(30 \mathrm{~mL})$, was added dropwise. With the water bath, the mixture was reacted at a temperature of $25^{\circ} \mathrm{C}$, for $5 \mathrm{~h}$, and then heated to reflux for $2 \mathrm{~h}$. After cooling to room temperature, the reaction mixture was filtered under vacuum and dried to produce ethyl 3-(3-substituted phenylureido)benzoates $\mathbf{1 a - 1 m}$, in yields ranging from $85 \%$ to $95 \%$.

\subsection{General Procedure for the Preparation of $\mathbf{2 a}-\mathbf{2} \mathbf{m}$}

Ethyl 3-(3-substituted phenylureido)benzoates $\mathbf{1 a}-\mathbf{1 m}(20 \mathrm{mmol})$ and $80 \%$ hydrazine hydrate $(100 \mathrm{mmol})$ were dissolved in ethanol $(100 \mathrm{~mL})$. The mixture was stirred and heated under reflux for $4 \mathrm{~h}$. After cooling to room temperature, the reaction mixture was filtered under vacuum. The solid residue was washed with ice-water $(50 \mathrm{~mL})$, to give 3-(3-substituted phenylureido)benzohydrazides $\mathbf{2 a}-\mathbf{2} \mathbf{m}$ in yields ranging from $80 \%$ to $95 \%$.

\subsection{General Procedure for the Preparation of $\mathbf{3 a}-\mathbf{3 i}$}

3-(3-Substituted phenylureido)benzohydrazides $\mathbf{2 a - 2 m}(4.42 \mathrm{mmol})$ was dissolved in dried tetrahydrofuran $(50 \mathrm{~mL})$ in a $250 \mathrm{~mL}$ round bottom flask. With ice water bath cooling, 2-chloronictinoyl chloride $(5.7 \mathrm{mmol})$ dissolved in dried tetrahydrofuran $(50 \mathrm{~mL})$, was added into the flask dropwise. The mixture was reacted at room temperature for $17 \mathrm{~h}$, then it was distilled under reduced pressure to remove the tetrahydrofuran. The solid residue was recrystallized from DMF and water (volume ratio, 1:1) to give the target compounds, $N^{\top}$-[3-(3-substituted phenylureido)benzoyl]- $N$ (2-chloro)nicotinohydrazides $\mathbf{3 a}-\mathbf{3 i}$, with yields ranging from $50 \%$ to $70 \%$.

\subsection{General Procedure for the Preparation of $\mathbf{4 a - 4 i}$}

3-(3-substituted phenylureido)benzohydrazides $\mathbf{2 a - 2 m ~ ( 2 ~} \mathrm{mmol})$ and 2-(4-nitrilo)benzyl-1-(3trifluoromethyl)phenyl ketones $(2 \mathrm{mmol})$ were dissolved in a mixture of methanol $(15 \mathrm{~mL})$ and $n$-hexane $(2 \mathrm{~mL})$ in a $100 \mathrm{~mL}$ round bottom flask and heated to $60{ }^{\circ} \mathrm{C}$ with a water bath. After adding three drops of trifluoroacetic acid, the mixture was stirred and reacted for $6 \mathrm{~h}$. The reaction mixture was filtered while hot and the solid obtained was washed with $15 \mathrm{~mL}$ methanol, then dried to give the target compounds, $\mathrm{N}$-(3-substituted phenylureido)benzoyl-1-(3-trifluoromethyl)phenyl-2-(4-cyano)-phenyl ethanone hydrazones $4 \mathbf{a}-\mathbf{4 i}$, in yields ranging from $60 \%$ to $75 \%$. All eighteen phenylurea derivatives 3a-3i, 4a-4i were novel and the physical and spectral data for these compounds are listed below.

$N^{\prime}-[3-(3-(2-M e t h y l)$ phenyl)ureido]benzoyl-N-(2-chloro)nicotinohydrazide (3a): Yellow solid, yield

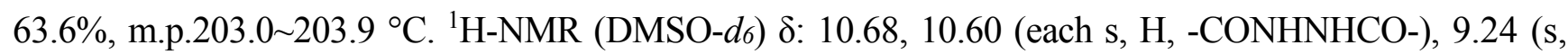
1H, NH), 8.55 (dd, $J=4.8,1.8 \mathrm{~Hz}, 1 \mathrm{H}), 7.96-8.02$ (overlap, 4H), 7.84 (d, $J=8.0 \mathrm{~Hz}, 1 \mathrm{H}$ ), 7.68 (d, $J=7.8 \mathrm{~Hz}, 1 \mathrm{H}), 7.58(\mathrm{dd}, J=7.4,4.9 \mathrm{~Hz}, 1 \mathrm{H}), 7.52(\mathrm{~d}, J=7.7 \mathrm{~Hz}, 1 \mathrm{H}), 7.42(\mathrm{t}, J=7.9 \mathrm{~Hz}, 1 \mathrm{H}), 7.19$ $(\mathrm{d}, J=7.4 \mathrm{~Hz}, 1 \mathrm{H}), 7.15(\mathrm{t}, J=7.6 \mathrm{~Hz}, 1 \mathrm{H}), 6.96(\mathrm{t}, J=7.4 \mathrm{~Hz}, 1 \mathrm{H}), 2.25\left(\mathrm{~s}, 3 \mathrm{H},-\mathrm{CH}_{3}\right) . \mathrm{IR}(\mathrm{KBr}): v$ $3281,3148,3027,1643,1588,1561,1455,1334,1295,1244,1182,994,752,693 \mathrm{~cm}^{-1}$. 
$N^{\prime}$-[3-(3-(2-Fluoro)phenyl)ureido]benzoyl-N-(2-chloro)nicotinohydrazide (3b): Gray white solid, yield $60.7 \%$, decomposed before melting. HR-ESI-MS m/z: $428.0908[\mathrm{M}+\mathrm{H}]^{+}$(calcd. for $\mathrm{C}_{20} \mathrm{H}_{17} \mathrm{ClFN}_{5} \mathrm{O}_{3}$, 428.0926). ${ }^{1} \mathrm{H}-\mathrm{NMR}$ (DMSO-d6) $\delta: 10.64$ (s, 2H, -CONHNHCO-), 9.29(s, 1H, NH), 8.62 (d, $J=2.1 \mathrm{~Hz}, 1 \mathrm{H}), 8.55(\mathrm{dd}, J=4.8,1.8 \mathrm{~Hz}, 1 \mathrm{H}), 8.16(\mathrm{dt}, J=8.2,1.3 \mathrm{~Hz}, 1 \mathrm{H}), 8.00(\mathrm{~d}, J=2.1 \mathrm{~Hz}, 1 \mathrm{H})$, $7.98(\mathrm{dd}, J=7.6,1.8 \mathrm{~Hz}, 1 \mathrm{H}), 7.67(\mathrm{dd}, J=8.0,1.8 \mathrm{~Hz}, 1 \mathrm{H}), 7.58(\mathrm{dd}, J=7.5,4.8 \mathrm{~Hz}, 1 \mathrm{H}), 7.55(\mathrm{~d}$, $J=7.8 \mathrm{~Hz}, 1 \mathrm{H}), 7.44(\mathrm{t}, J=7.9 \mathrm{~Hz}, 1 \mathrm{H}), 7.24(\mathrm{ddd}, J=11.5,8.2,1.1 \mathrm{~Hz}, 1 \mathrm{H}), 7.15(\mathrm{t}, J=7.7 \mathrm{~Hz}, 1 \mathrm{H})$, 7.03 (ddt, $J=7.3,5.2,1.5 \mathrm{~Hz}, 1 \mathrm{H})$. IR (KBr): v 3566, 3394, 3297, 3211, 3078, 3011, 1647, 1588, $1561,1491,1451,1405,1299,1252,1193,806,752,646 \mathrm{~cm}^{-1}$.

$N^{\prime}-[3-(3-(2,4-D i f l u o r o)$ phenyl)ureido]benzoyl-N-(2-chloro)nicotinohydrazide (3c): Gray white solid, yield 58.3\%, m.p. $184.2 \sim 185.9{ }^{\circ} \mathrm{C}$. HR-ESI-MS $m / z$ : $446.0808[\mathrm{M}+\mathrm{H}]^{+}$(calcd. for $\mathrm{C}_{20} \mathrm{H}_{15} \mathrm{ClF}_{2} \mathrm{~N}_{5} \mathrm{O}_{3}$, 446.0826). ${ }^{1} \mathrm{H}-\mathrm{NMR}$ (DMSO-d6) $\delta: 10.68,10.60$ (each s, $1 \mathrm{H}$, -CONHNHCO-), 9.21 (s, 1H, NH), 8.56 $(\mathrm{d}, J=2.1 \mathrm{~Hz}, 1 \mathrm{H}), 8.55(\mathrm{dd}, J=4.8,1.9 \mathrm{~Hz}, 1 \mathrm{H}), 8.09(\mathrm{dt}, J=9.2,6.1 \mathrm{~Hz}, 1 \mathrm{H}), 7.98(\mathrm{~s}, 1 \mathrm{H}), 7.95$ (s, 1H), $7.66(\mathrm{dd}, J=8.0,1.3 \mathrm{~Hz}, 1 \mathrm{H}), 7.57$ (dd, $J=7.5,4.8 \mathrm{~Hz}, 1 \mathrm{H}), 7.55$ (d, $J=7.8 \mathrm{~Hz}, 1 \mathrm{H}), 7.43$ (t, $J=7.9 \mathrm{~Hz}, 1 \mathrm{H}), 7.33$ (ddd, $J=11.6,8.9,2.8 \mathrm{~Hz}, 1 \mathrm{H}), 7.06$ (t, $J=7.8 \mathrm{~Hz}, 1 \mathrm{H})$. IR (KBr): v 3539, 3304, $3172,3035,1721,1663,1600,1553,1432,1401,1190,1139,1096,845,802,740 \mathrm{~cm}^{-1}$.

$N^{\prime}$-[3-(3-(3,4-Difluoro)phenyl)ureido]benzoyl-N-(2-chloro)nicotinohydrazide (3d): Yellow solid, yield 52.5\%, m.p. 214.9 215.2 ${ }^{\circ} \mathrm{C}$. HR-ESI-MS $m / z$ : $446.0829[\mathrm{M}+\mathrm{H}]^{+}$(calcd. for $\mathrm{C}_{20} \mathrm{H}_{15} \mathrm{ClF}_{2} \mathrm{~N}_{5} \mathrm{O}_{3}, 446.0826$ ). ${ }^{1} \mathrm{H}-\mathrm{NMR}$ (DMSO-d6) $\delta$ : 10.67, 10.59 (each s, 1H, -CONHNHCO-), 8.97 (s, 2H, -NHCONH-), 8.55 (dd, $J=4.8,1.8 \mathrm{~Hz}, 1 \mathrm{H}$ ), 7.96-8.02 (overlap, 2H), 7.64-7.70 (overlap, 2H), 7.58 (dd, $J=7.5,4.8 \mathrm{~Hz}$, $1 \mathrm{H}), 7.55$ (d, $J=7.8 \mathrm{~Hz}, 1 \mathrm{H}), 7.43$ (t, $J=7.9 \mathrm{~Hz}, 1 \mathrm{H}), 7.36$ (d, $J=10.4 \mathrm{~Hz}, 1 \mathrm{H}), 7.15$ (m, 1H). IR (KBr): $v 3344,3249,3091,1685,1648,1561,1515,1490,1436,1403,1208,905,860,748,673 \mathrm{~cm}^{-1}$.

N'-[3-(3-(3,4-Dichloro)phenyl)ureido]benzoyl-N-(2-chloro)nicotinohydrazide (3e): Gray white solid, yield 54.9\%, m.p. 194.2 194.7 ${ }^{\circ} \mathrm{C}$. HR-ESI-MS m/z: $478.0235[\mathrm{M}+\mathrm{H}]^{+}$(calcd. for $\mathrm{C}_{20} \mathrm{H}_{15} \mathrm{Cl}_{3} \mathrm{~N}_{5} \mathrm{O}_{3}$, 478.0235). ${ }^{1} \mathrm{H}-\mathrm{NMR}$ (DMSO-d6) $\delta: 10.68,10.60$ (each s, 1H, -CONHNHCO-), 9.06, 9.02 (each s, 1H, -NHCONH-), 8.55 (dd, $J=4.8,1.9 \mathrm{~Hz}, 1 \mathrm{H}), 8.01$ (s, 1H), 7.99 (dd, $J=7.5,1.9 \mathrm{~Hz}, 1 \mathrm{H}), 7.91$ (d, $J=2.4 \mathrm{~Hz}, 1 \mathrm{H}), 7.66(\mathrm{~d}, J=8.0 \mathrm{~Hz}, 1 \mathrm{H}), 7.58(\mathrm{dd}, J=7.6,4.8 \mathrm{~Hz}, 1 \mathrm{H}), 7.56(\mathrm{~d}, J=7.8 \mathrm{~Hz}, 1 \mathrm{H}) 7.53$ $(\mathrm{d}, J=8.8 \mathrm{~Hz}, 1 \mathrm{H}), 7.43(\mathrm{t}, J=7.9 \mathrm{~Hz}, 1 \mathrm{H}), 7.35$ (dd, $J=8.8,2.4 \mathrm{~Hz}, 1 \mathrm{H}) . \mathrm{IR}(\mathrm{KBr}): v 3277,3236$, $3093,3010,1653,1581,1536,1473,1401,1378,1292,1223,1132,906,808,748 \mathrm{~cm}^{-1}$.

$N^{\prime}$-[3-(3-(3,5-Dichloro)phenyl)ureido]benzoyl-N-(2-chloro)nicotinohydrazide (3f): Gray white solid, yield 65.6\%, m.p. 223.6 224.5 ${ }^{\circ} \mathrm{C}$. HR-ESI-MS m/z: $478.0235[\mathrm{M}+\mathrm{H}]^{+}$(calcd. for $\mathrm{C}_{20} \mathrm{H}_{15} \mathrm{Cl}_{3} \mathrm{~N}_{5} \mathrm{O}_{3}$, 478.0235). ${ }^{1} \mathrm{H}-\mathrm{NMR}$ (DMSO-d $\left.d_{6}\right) \delta: 10.68,10.60$ (each s, 1H, -CONHNHCO-), 9.12, 9.11 (each s, $1 \mathrm{H}$, -NHCONH-), 8.56 (dd, $J=4.8,1.9 \mathrm{~Hz}, 1 \mathrm{H}), 8.01$ (s, 1H), 7.99 (dd, $J=7.5,1.8 \mathrm{~Hz}, 1 \mathrm{H}), 7.95$ (s, 1H), 7.66 (d, $J=7.9 \mathrm{~Hz}, 1 \mathrm{H}), 7.54-7.60$ (overlap, 3H), 7.45 (t, $J=7.9 \mathrm{~Hz}, 1 \mathrm{H}), 7.17$ (t, $J=1.8 \mathrm{~Hz}, 1 \mathrm{H})$. IR (KBr): v 3362, 3308, 3168, 1725, 1659, 1604, 1584,1538, 1484, 1392, 1304, 1281, 1246, 1195, 1100 , $1063,820,739,665 \mathrm{~cm}^{-1}$.

N'-[3-(3-(4-Ditrifluoromethylmethyl)phenyl)ureido]benzoyl-N-(2-chloro)nicotinohydrazide (3g): white powder, yield 62.4\%, m.p. 196.76 198.1 ${ }^{\circ} \mathrm{C} .{ }^{1} \mathrm{H}-\mathrm{NMR}$ (DMSO-d6) $\delta: 10.64$ (s, 2H, -CONHNHCO-), 9.45 (s, 1H, NH), 8.55 (dd, $J=4.8,1.8 \mathrm{~Hz}, 1 \mathrm{H}), 8.24$ (d, $J=8.8 \mathrm{~Hz}, 1 \mathrm{H}), 8.22(\mathrm{~s}, 1 \mathrm{H}), 8.02(\mathrm{~s}, 1 \mathrm{H})$, 
7.99 (dd, $J=7.5,1.8 \mathrm{~Hz}, 1 \mathrm{H}), 7.69$ (dd, $J=7.9,1.2 \mathrm{~Hz}, 1 \mathrm{H}), 7.58$ (dd, $J=7.5,4.8 \mathrm{~Hz}, 1 \mathrm{H}), 7.55$ (d, $J=7.8 \mathrm{~Hz}, 1 \mathrm{H}$ ), 7.43-7.49 (overlap, 3H), 2.35 (s, 3H, - $\mathrm{PhCH}_{3}$ ). IR (KBr): v 3254, 3005, 1662, 1613, $1584,1533,1401,1306,1272,1218,1178,1135,1106,977,888,811,754 \mathrm{~cm}^{-1}$.

$N^{\prime}-[3-(3-(2-T r i f l u o r o m e t h y l)$ phenyl)ureido]benzoyl-N-(2-chloro)nicotinohydrazide (3h): Yellow solid, yield 68.7\%, m.p. $199.3 \sim 199.6{ }^{\circ} \mathrm{C}$. HR-ESI-MS m/z: $478.0889[\mathrm{M}+\mathrm{H}]^{+}$(calcd. for $\mathrm{C}_{21} \mathrm{H}_{16} \mathrm{ClF}_{3} \mathrm{~N}_{5} \mathrm{O}_{3}$, 478.0888). ${ }^{1} \mathrm{H}-\mathrm{NMR}$ (DMSO-d6) $\delta: 10.66,10.62$ (each s, $1 \mathrm{H}$, -CONHNHCO-), 9.58 (s, 1H, NH), 8.55 (dd, $J=4.8,1.2 \mathrm{~Hz}, 1 \mathrm{H}), 8.13(\mathrm{~s}, 1 \mathrm{H}), 7.94-8.02$ (m, overlap, 2H), 7.62-7.72 (m, overlap, 3H), 7.53-7.60 (m, overlap, 2H), $7.434(\mathrm{t}, J=7.9 \mathrm{~Hz}, 1 \mathrm{H}), 7.30$ (t, $J=7.5 \mathrm{~Hz}, 2 \mathrm{H})$. IR (KBr): v 3572, 3435, $3303,3216,3083,3012$, 1652, 1590, 1544, 1507, 1453, 1407, 1316, 1279, 1179, 1109, 1059, 1038, $905,806,764,690,648 \mathrm{~cm}^{-1}$.

$N^{\prime}$-[3-(3-(4-Trifluoromethoxyl)phenyl)ureido]benzoyl-N-(2-chloro)nicotinohydrazide $\quad$ (3i): $\quad$ white powder, yield 59.1\%, m.p. 233.6 234.4 ${ }^{\circ} \mathrm{C}$. HR-ESI-MS m/z: $494.0839[\mathrm{M}+\mathrm{H}]^{+}$(calcd. for $\mathrm{C}_{21} \mathrm{H}_{16} \mathrm{ClF}_{3} \mathrm{~N}_{5} \mathrm{O}_{4}, 494.0837$ ). ${ }^{1} \mathrm{H}-\mathrm{NMR}$ (DMSO- $d_{6}$ ) $\delta: 10.67,10.60$ (s, 1H, -CONHNHCO-), 8.99, 8.97 (each s, 1H, -NHCONH-), 8.55 (dd, $J=4.8,1.8 \mathrm{~Hz}, 1 \mathrm{H}), 7.97-8.02$ (m, overlap, 2H), 7.94 (s, 1H), $7.67(\mathrm{~d}, J=7.7 \mathrm{~Hz}, 1 \mathrm{H}), 7.58(\mathrm{~d}, J=8.6 \mathrm{~Hz}, 1 \mathrm{H}), 7.54(\mathrm{~d}, J=7.3 \mathrm{~Hz}, 1 \mathrm{H}), 7.43$ (t, $J=7.9 \mathrm{~Hz}, 1 \mathrm{H})$, $7.30(\mathrm{~d}, J=8.7 \mathrm{~Hz}, 2 \mathrm{H})$. IR (KBr): $v$ 3373, 3299, 3182, 3033, 2929, 2855, 1723, 1660, 1611, 1590, $1507,1478,1399,1279,1154,1096,827,744,682,636 \mathrm{~cm}^{-1}$.

N-[3-(3-(2-Methyl)phenyl)ureido]benzoyl-1-(3-trifluoromethyl)phenyl-2-(4-cyano)phenylethanone hydrazone (4a): White powder, yield 70.0\%, m.p. 208.3 208.7 ${ }^{\circ} \mathrm{C}$. HR-ESI-MS $m / z: 556.195[\mathrm{M}+\mathrm{H}]^{+}$ (calcd for $\mathrm{C}_{31} \mathrm{H}_{25} \mathrm{~F}_{3} \mathrm{~N}_{5} \mathrm{O}_{2}, 556.1955$ ). ${ }^{1} \mathrm{H}-\mathrm{NMR}$ (DMSO-d6) $\delta$ : 11.34 (s, 1H, PhCONH-), 9.24 (s, 1H, -NHCONH-), 8.17 (s, 1H), 8.07 (s, 1H), $7.96(\mathrm{~s}, 1 \mathrm{H}), 7.91(\mathrm{~s}, 1 \mathrm{H}), 7.83(\mathrm{~d}, J=7.7 \mathrm{~Hz}, 1 \mathrm{H}), 7.77$ $(\mathrm{d}, J=8.0 \mathrm{~Hz}, 2 \mathrm{H}), 7.73$ (s, 1H), 7.55-7.70 (overlap, 2H), 7.38-7.45 (overlap, 3H), 7.34 (d, $J=7.7 \mathrm{~Hz}$, 1H), 7.12-7.22 (overlap, 2H), 6.96 (t, $J=7.2 \mathrm{~Hz}, 1 \mathrm{H}), 4.60$ (s, 2H, - $\left.\mathrm{CH}_{2}-\right), 2.24$ (s, 3H, - $\left.\mathrm{CH}_{3}\right)$. IR (KBr):v 3336, 3307, 3216, 3050, 2958, 2229, 1652, 1590, 1548, 1486, 1432, 1349, 1258, 1171, 1117 , $1080,893,764,702,644,545 \mathrm{~cm}^{-1}$.

N-[3-(3-(2-Fluoro)phenyl)ureido]benzoyl-1-(3-trifluoromethyl)phenyl-2-(4-cyano)phenylethanone hydrazone (4b): White powder, yield 63.4\%, m.p. 239.0 239.6 ${ }^{\circ} \mathrm{C}$. HR-ESI-MS $\mathrm{m} / \mathrm{z}: 560.1706$ $[\mathrm{M}+\mathrm{H}]^{+}$(calcd for $\left.\mathrm{C}_{30} \mathrm{H}_{22} \mathrm{~F}_{3} \mathrm{~N}_{5} \mathrm{O}_{2}, 560.1704\right) .{ }^{1} \mathrm{H}-\mathrm{NMR}$ (DMSO-d6) $\delta: 11.34$ (s, 1H, PhCONH-), 9.27, 8.59 (each s, 1H, -NHCONH-), 8.15 (t, $J=8.0 \mathrm{~Hz}, 2 \mathrm{H}), 8.07$ (s, 1H), 7.92 (s, 1H), 7.77 (d, $J=8.2 \mathrm{~Hz}$, 2H), $7.72(\mathrm{~s}, 1 \mathrm{H}), 7.63(\mathrm{~s}, 2 \mathrm{H}), 7.35-7.45(\mathrm{~m}$, overlap, 4H), $7.25(\mathrm{dd}, J=10.7,8.4 \mathrm{~Hz}, 1 \mathrm{H}), 7.15(\mathrm{t}$, $J=7.4 \mathrm{~Hz}, 1 \mathrm{H}), 7.01$ (dd, $J=12.6,6.2 \mathrm{~Hz}, 1 \mathrm{H}), 4.60$ (s, 2H, - $\mathrm{CH}_{2}-$ ). IR (KBr): $v$ 3328, 3257, 3104, 2925, 2224, 1723, 1669, 1590, 1544, 1519, 1436, 1295, 1129, 1071, 752, 694, $553 \mathrm{~cm}^{-1}$.

N-[3-(3-(2,4-Difluoro)phenyl)ureido]benzoyl-1-(3-trifluoromethyl)phenyl-2-(4-cyano)phenylethanone hydrazone (4c): White powder, yield 67.5\%, m.p. 243.6 243.7 ${ }^{\circ} \mathrm{C}$. HR-ESI-MS $m / z: 578.1612[\mathrm{M}+\mathrm{H}]^{+}$ (calcd for $\mathrm{C}_{30} \mathrm{H}_{21} \mathrm{~F}_{5} \mathrm{~N}_{5} \mathrm{O}_{2}, 578.1610$ ). ${ }^{1} \mathrm{H}-\mathrm{NMR}$ (DMSO-d6) $\delta: 11.33$ (s, 1H, PhCONH-), 9.21, 8.54 (each s, 1H, -NHCONH-), 8.17 (s, 1H), 8.02-8.12 (overlap, 2H), 7.91 (s, 1H), 7.77 (d, J=8.2 Hz, 2H), $7.73(\mathrm{~s}, 1 \mathrm{H}), 7.63(\mathrm{~s}, 2 \mathrm{H}), 7.35-7.45$ (overlap, 4H), $7.30(\mathrm{~m}, 1 \mathrm{H}), 7.06(\mathrm{t}, J=8.2 \mathrm{~Hz}, 1 \mathrm{H}), 4.60$ (s, 2H, 
$-\mathrm{CH}_{2}-$ ). IR (KBr): v 3535, 3308, 3257, 3187,2577, 1678, 1565, 1397, 1186, 1131, 1096, 1064, 842, $744,611 \mathrm{~cm}^{-1}$.

N-[3-(3-(3,4-Difluoro)phenyl)ureido]benzoyl-1-(3-trifluoromethyl)phenyl-2-(4-cyano)phenylethanone hydrazone (4d): White powder, yield 69.6\%, m.p. 243.2 243.5 ${ }^{\circ} \mathrm{C}$. HR-ESI-MS $\mathrm{m} / \mathrm{z}: 578.1606$ $[\mathrm{M}+\mathrm{H}]^{+}$(calcd for $\left.\mathrm{C}_{30} \mathrm{H}_{21} \mathrm{~F}_{5} \mathrm{~N}_{5} \mathrm{O}_{2}, 578.1610\right) .{ }^{1} \mathrm{H}-\mathrm{NMR}$ (DMSO-d6) $\delta: 11.32$ (s, 1H, PhCONH-), 8.96, 8.94 (each s, 1H, -NHCONH-), 8.17 (s, 1H), 8.06 (s, 1H), 7.90 (s, 1H), 7.77 (d, J=8.2 Hz, 2H), 7.73 (s, 1H), 7.59-7.70 (m, overlap, 3H), 7.30-7.45 (m, overlap, 5H), 7.14 (d, J=7.9 Hz, 1H), 4.60 (s, 2H, - $\mathrm{CH}_{2-}$ ). IR (KBr): v3336, 3316, 3258, 3101, 2226, 1718, 1663, 1593, 1550, 1510, 1432, 1209, 1131 , $1072,1022,869,689,552 \mathrm{~cm}^{-1}$.

N-[3-(3-(3,4-Dichloro)phenyl)ureido]benzoyl-1-(3-trifluoromethyl)phenyl-2-(4-cyano)phenylethanone hydrazone (4e): White powder, yield 72.4\%, m.p. 245.4 245.8 ${ }^{\circ} \mathrm{C}$. HR-ESI-MS $m / z: 610.1021[\mathrm{M}+\mathrm{H}]^{+}$ (calcd. for $\mathrm{C}_{30} \mathrm{H}_{21} \mathrm{Cl}_{2} \mathrm{~F}_{3} \mathrm{~N}_{5} \mathrm{O}_{2}, 610.1019$ ). ${ }^{1} \mathrm{H}-\mathrm{NMR}$ (DMSO-d6) $\delta: 11.33$ (s, 1H, PhCONH-), 9.07, 9.05 (each s, 1H, -NHCONH-), $8.18(\mathrm{~s}, 1 \mathrm{H}), 8.06(\mathrm{~s}, 1 \mathrm{H}), 7.92(\mathrm{~s}, 1 \mathrm{H}), 7.89(\mathrm{~d}, J=2.2 \mathrm{~Hz}, 1 \mathrm{H}), 7.77(\mathrm{~d}$, $J=8.2 \mathrm{~Hz}, 2 \mathrm{H}), 7.73(\mathrm{~s}, 1 \mathrm{H}), 7.64(\mathrm{~s}, 2 \mathrm{H}), 7.52$ (d, $J=8.8 \mathrm{~Hz}, 1 \mathrm{H}), 7.31-7.45$ (m, overlap, 5H), 4.60 (s, 2H, - $\mathrm{CH}_{2}$ ). IR (KBr): v 3325, 3299, 3096, 3033, 2228, 1716, 1659, 1590, 1518, 1473, 1430, 1378, $1326,1289,1192,1132,1069,854,811,745,691,545 \mathrm{~cm}^{-1}$.

N-[3-(3-(2,6-Dimethyl)phenyl)ureido]benzoyl-1-(3-trifluoromethyl)phenyl-2-(4-cyano)phenyl ethanone hydrazone (4f): White powder, yield 70.4\%, m.p. 240.1 240.3 ${ }^{\circ} \mathrm{C} .{ }^{1} \mathrm{H}-\mathrm{NMR}$ (DMSO-d6) $\delta: 11.31$ (s, 1H, PhCONH-), 8.96 (s, 1H, -NHCONH-), 8.16 (s, 1H), 8.06 (s, 1H), 7.91 (s, 1H), 7.77 (s, 1H), 7.75 (s, 1H), 7.73 (s, 1H), 7.56-7.69 (overlap, 2H), 7.39 (d, J=8.1 Hz, 2H), 7.36 (d, J = 7.9 Hz, 1H), 7.31 (d, $J=7.7 \mathrm{~Hz}, 1 \mathrm{H}), 7.05-7.11$ (overlap, 3H), 4.58 (s, 2H, - $\left.\mathrm{CH}_{2}-\right), 2.20$ (s, 6H, - $\mathrm{CH}_{3}$ ). IR (KBr): v 3295, $3159,3004,2227,1655,1640,1607,1595,1487,1342,1307,1266,1241,1176,800,770,745 \mathrm{~cm}^{-1}$.

N-[3-(3-(3-Trifluoromethyl)phenyl)ureido]benzoyl-1-(3-trifluoromethyl)phenyl-2-(4-cyano)phenyl ethanone hydrazone (4g): White powder, yield 68.7\%, m.p. 220.0 221.2 ${ }^{\circ} \mathrm{C}$. HR-ESI-MS $\mathrm{m} / \mathrm{z}$ : $610.1669[\mathrm{M}+\mathrm{H}]^{+}$(calcd. for $\mathrm{C}_{31} \mathrm{H}_{22} \mathrm{~F}_{6} \mathrm{~N}_{5} \mathrm{O}_{2}, 610.1672$ ). ${ }^{1} \mathrm{H}-\mathrm{NMR}$ (DMSO-d6) $\delta: 11.33(\mathrm{~s}, 1 \mathrm{H}$, PhCONH-), 9.08, 9.01 (each s, 1H, -NHCONH-), 8.18 (s, 1H), 8.07 (s, 1H), 8.03 (s, 1H), 7.93 (s, 1H), $7.77(\mathrm{~d}, J=8.2 \mathrm{~Hz}, 2 \mathrm{H}), 7.73$ (s, 1H), 7.61-7.70 (overlap, 2H), 7.59 (d, $J=8.0 \mathrm{~Hz}, 1 \mathrm{H}), 7.52$ (t, $J=7.9 \mathrm{~Hz}$, 1H), 7.35-7.45 (m, overlap, 4H), 7.32 (d, $J=7.6 \mathrm{~Hz}, 1 \mathrm{H}), 4.60$ (s, 2H, - $\mathrm{CH}_{2}$ ). IR (KBr): v3336, 3261, $3207,3093,2225,1717,1663,1596,1553,1514,1491,1444,1330,1295,1252,1197,1154,1115$, $1068,869,795,752,693,670,552 \mathrm{~cm}^{-1}$.

N-[3-(3-(3-Fluoro)phenyl)ureido]benzoyl-1-(3-trifluoromethyl)phenyl-2-(4-cyano)phenylethanone hydrazone (4h): Yellow powder, yield 62.5\%, m.p. 231.7 231.9 ${ }^{\circ} \mathrm{C}$. HR-ESI-MS m/z: 560.1706 $[\mathrm{M}+\mathrm{H}]^{+}$(calcd. for $\left.\mathrm{C}_{30} \mathrm{H}_{22} \mathrm{~F}_{4} \mathrm{~N}_{5} \mathrm{O}_{2}, 560.1704\right) .{ }^{1} \mathrm{H}-\mathrm{NMR}$ (DMSO-d $)$ ) $\delta: 11.33$ (s, 1H, PhCONH-), 8.94 (s, 2H, -NHCONH-), 8.17, 8.07, 7.91 (each s, 1H), 7.77 (d, $J=8.2 \mathrm{~Hz}, 2 \mathrm{H}), 7.73,7.65$ (each s, 1H), 7.50 (d, $J=11.8 \mathrm{~Hz}, 1 \mathrm{H}), 7.35-7.45$ (m, overlap, 4H), 7.31 (dd, $J=15.3,7.9 \mathrm{~Hz}, 1 \mathrm{H}), 7.14(\mathrm{~d}, J=7.8 \mathrm{~Hz}), 6.79$ (dt, $J=8.4,1.9 \mathrm{~Hz}, 1 \mathrm{H}), 4.60$ (s, 2H, - $\mathrm{CH}_{2}-$ ). IR (KBr): v3340, 3311, 3257, 3145, 3100, 2224, 1714, $1664,1511,1490,1436,1308,1196,1071,1017,897,872,752,690,557 \mathrm{~cm}^{-1}$. 
N-[3-(3-(3-Bromo)phenyl)ureido]benzoyl-1-(3-trifluoromethyl)phenyl-2-(4-cyano)phenyl ethanone hydrazone (4i): White powder, yield 61.8\%, m.p. 232.4 232.8 ${ }^{\circ} \mathrm{C}$. HR-ESI-MS $m / z: 620.0906[\mathrm{M}+\mathrm{H}]^{+}$ (calcd. for $\mathrm{C}_{30} \mathrm{H}_{22} \mathrm{BrF}_{3} \mathrm{~N}_{5} \mathrm{O}_{2}, 620.0903$ ). ${ }^{1} \mathrm{H}-\mathrm{NMR}$ (DMSO-d6) $\delta: 11.33$ (s, 1H, PhCONH-), 8.97, 8.93 (each s, 1H, -NHCONH-), 8.18, 8.07, 7.92 (each s, 1H), 7.86 (s, 1H), 7.77 (d, J=8.2 Hz, 2H), 7.74 (s, 1H), 7.64 (s, 2H), 7.39-7.44 (m, overlap, 3H), 7.37 (d, $J=7.6 \mathrm{~Hz}, 1 \mathrm{H}), 7.33$ (d, $J=7.4 \mathrm{~Hz}, 1 \mathrm{H}), 7.25$ (t, $J=8.0 \mathrm{~Hz}$, $1 \mathrm{H}), 7.16(\mathrm{~d}, J=7.7 \mathrm{~Hz}, 1 \mathrm{H}), 4.60$ (s, 2H, $\left.-\mathrm{CH}_{2-}\right)$. IR (KBr): v3336, 3303, 3265, 3104, 2224, 1723, 1656, 1590, 1536, 1515, 1478, 1428, 1300, 1196, 1129, 1071, 1017, 993, 901, 856, 752, 694, $553 \mathrm{~cm}^{-1}$.

\subsection{Bioassay for Insecticidal Activities}

Wheat leaf discs $(0.5 \mathrm{~cm} \times 0.5 \mathrm{~cm})$ were treated with $5 \mu \mathrm{L}$ solutions made from $5 \mathrm{mg}$ of test sample dissolved in $500 \mathrm{~mL}$ of acetone. Tebufenozide, chlorbenzuron and metaflumizone were used as positive controls and acetone was used as negative control. The third instars larvae of beet armyworm were fed with the discs. Cohorts of 24 beet armyworms were treated each time and bioassays were replicated three times. After $24 \mathrm{~h}, 48 \mathrm{~h}$ and $72 \mathrm{~h}$, the numbers of knocked down larvae (symptoms: the larvae were narcotized, the bodies were very soft and immobilized, and response disappeared completely) were recorded respectively [16,17].

Bioassay for insecticidal activities against cotton bollworm, diamondback moth and cabbage worm was performed according to the above method. The third instars larvae of cotton bollworm, diamondback moth and cabbage worm were fed with the discs instead, respectively. The numbers of knocked down larvae after $72 \mathrm{~h}$ were recorded. The experiments showed that the mortality of acetone as negative controls was $0 \%$.

\section{Conclusions}

In conclusion, eighteen phenylurea derivatives $\mathbf{3 a}-\mathbf{3 i}, \mathbf{4 a}-\mathbf{4 i}$ have been designed and synthesized according to the method of active groups linkage and the principle of aromatic groups bioisosterism. The insecticidal activities of these novel phenylurea derivatives against the third instars larvae of $S$. exigua, H. armigera, $P$. xyllostella and $P$. rapae were evaluated, at a concentration of $10 \mathrm{mg} / \mathrm{L}$. The bioassay results showed that all of the synthesized compounds displayed high insecticidal activity. Most of them presented higher insecticidal activity against $S$. exigua than the reference compounds tebufenozide, chlorbenzuron and metaflumizone. Among the synthesized compounds, 3b, 3d, 3f, 4b and $\mathbf{4 g}$ displayed broad spectrum insecticidal activity. The results above have encouraged us to further explore novel phenylurea derivatives as insecticidal agents and this will be reported in future work.

\section{Supplementary Materials}

Supplementary materials can be accessed at: http://www.mdpi.com/1420-3049/20/03/5050/s1.

\section{Acknowledgments}

This work was supported by Wheat Innovation Team of Modern Agricultural Industry Technology System of Shandong Province and High Level Talents Fund of Qingdao Agricultural University (No. 6631110). 


\section{Author Contributions}

Jialong Sun designed the research; Jialong Sun and Yuanming Zhou performed the research and analyzed the data; Yuanming Zhou wrote the paper. All authors read and approved the final manuscript.

\section{Conflicts of Interest}

The authors declare no conflict of interest.

\section{References}

1. Zhang, J.; Tang, X.H.; Ishaaya, I.; Cao, S.; Wu, J.J.; Yu, J.L.; Li, H.; Qian, X.H. Synthesis and insecticidal activity of heptafluoroisopropyl-containing benzoylphenylurea structures. J. Agric. Food Chem.2010, 58, 2736-2740.

2. Oberlander, H.; Silhacek, D.L. Mode of action of insect growth regulators in lepidopteran tissue culture. Pestic. Sci.1998, 54, 300-302.

3. Sun, R.F.; Zhang, Y.L.; Bi, F.C.; Wang, Q.M. Design, synthesis, and bioactivity study of novel benzoylpyridazyl ureas. J. Agric. Food Chem.2009, 57, 6356-6361.

4. Sun, R.F.; Lu, M.Y.; Chen, L.; Li Q.S.; Song, H.B.; Bi, F.C.; Huang, R.Q.; Wang, Q.M. Design, synthesis, bioactivity, and structure-activity relationship (SAR) studies of novel benzoylphenylureas containing oxime ether group. J. Agric. Food Chem. 2008, 56, 11376-11391.

5. Sun, R.F.; Liu, Y.X.; Zhang, Y.L.; Xiong, L.X.; Wang, Q.M. Design and synthesis of benzoylphenylureas with fluorinated substituents on the aniline ring as insect growth regulators. J. Agric. Food Chem. 2011, 59, 2471-2477.

6. Wing, K.D. RH-5849, a nonsteroidal ecdysone agonist: Effects on adrosophila cell line. Science 1988, 241, 467-469.

7. Sun, R.F.; Zhang, Y.L.; Chen, L.; Li, Y.Q.; Li, Q.S.; Song, H.B.; Huang, R.Q.; Bi, F.C.; Wang, Q.M. Design, synthesis, and insecticidal activities of new $N$-benzoyl- $N$ '-phenyl- $N$ '-sulfenylureas. J. Agric. Food Chem.2009, 57, 3661-3668.

8. Zhao, P.L.; Li, J.; Yang, G.F. Synthesis and insecticidal activity of chromanone and chromone analogues of diacylhydrazines. Bioorg. Med. Chem. 2007, 15, 1888-1895.

9. Sawada, Y.; Yanai, T.; Nakagawa, H.; Tsukamoto, Y.; Yokoi, S.; Yanagi, M.; Toya, T.; Sugizaki, H.; Kato, Y.; Shirakura, H.; et al. Synthesis and insecticidal activity of benzoheterocyclic analogues of $N^{\prime}$-benzoyl- $N$-(tert-butyl) benzohydrazide: Part 1. Design of benzoheterocyclic analogues. Pest Manag. Sci. 2003, 59, 25-35.

10. Dhadialla, T.S.; Jansson, R.K. Non-steroidal ecdysone agonists: new tools for IPM and insect resistance management. Pestic. Sci.1999, 55, 357-359.

11. Wang, H.; Yang, Z.K.; Fan, Z.J.; Wu, Q.J.; Zhang, Y.J.; Mi, A.; Wang, S.X.; Zhang, Z.C.; Song, H.B.; Liu, F. Synthesis and insecticidal activity of $N$-tert-butyl- $N, N$-diacylhydrazines containing 1,2,3-thiadiazoles. J. Agric. Food Chem. 2011, 59, 628-634.

12. Chatterjee N.S.; Gupta, S.; Varghese, E. Degradation of metaflumizone in soil: Impact of varying moisture, light, temperature, atmospheric $\mathrm{CO}_{2}$ level, soil type and soil sterilization. Chemosphere 2013, 90, 729-736. 
13. Salgado, V.L.; Hayashi, J.H. Metaflumizone is a novel sodium channel blocker insecticide. Vet. Parasitol. 2007, 150, 182-189.

14. Hempel, K.; Hess, F.G.; Bogi, C.; Fabian, E.; Hellwig, J.; Fegert, I. Toxicological properties of metaflumizone. Vet. Parasitol.2007, 150, 190-195.

15. Li, Y.Q.; Qin, Y.G.; Yang, N.; Sun, Y.F.; Yang, X.L.; Sun, R.F.; Wang, Q.M.; Ling, Y. Studies on insecticidal activities and action mechanism of novel benzoylphenylurea candidate NK-17. PLoS One 2013, 8, e66251.

16. Mao, C.H.; Wang, Q.M.; Huang, R.Q.; Bi, F.C.; Chen, L.; Liu, Y.X.; Shang, J. Synthesis and insecticidal evaluation of novel $N$-oxalyl derivatives of tebufenozide. J. Agric. Food Chem. 2004, $52,6737-6741$.

17. Zhang, J.W.; Hu, Z.; Li, S.K.; Wu, W.J. Synthesis and insecticidal activities of new ester-derivatives of Celangulin-V. Int. J. Mol. Sci. 2011, 12, 9596-9604.

Sample Availability: Samples of the compounds $\mathbf{3 a}-\mathbf{3 i}, \mathbf{4 a}-\mathbf{4 i}$ are available from the authors.

(C) 2015 by the authors; licensee MDPI, Basel, Switzerland. This article is an open access article distributed under the terms and conditions of the Creative Commons Attribution license (http://creativecommons.org/licenses/by/4.0/). 